\title{
Gremlin1 Delivered by Mesenchymal Stromal Cells Promoted Epithelial- Mesenchymal Transition in Human Esophageal Squamous Cell Carcinoma
}

\author{
Dongxi Hong ${ }^{a, b}$ Te Liuc Weijun Huanga,b Yan Liao ${ }^{a, b} \quad$ Lin Wang $^{\mathrm{a}}$ Zhen Zhang ${ }^{\mathrm{a}, \mathrm{b}}$ \\ Haixuan Chen ${ }^{e}$ Xinchun Zhang ${ }^{f}$ Qiuling Xiang ${ }^{a, b}$ \\ ${ }^{a}$ Center for Stem Cell Biology and Tissue Engineering, Key Laboratory for Stem Cells and Tissue \\ Engineering, Ministry of Education, Sun Yat-Sen University, Guangzhou, ${ }^{b} Z$ hongshan School of \\ Medicine, Sun Yat-Sen University, Guangzhou, 'Central Laboratory, Shanghai Geriatric Institute of \\ Chinese Medicine, Shanghai University of Traditional Chinese Medicine, Shanghai, dDepartment \\ of Pathology, Sun Yat-Sen Memorial Hospital, Sun Yat-Sen University, Guangzhou, Translational \\ Medicine Center, the First Affiliated Hospital of Sun Yat-Sen University, Guangzhou, 'Department of \\ Prosthodontics, Guanghua School of Stomatology \& Hospital of Stomatology, Guangdong Provincial \\ Key Laboratory of Stomatology, Sun Yat-Sen University, Guangzhou, China
}

\section{Key Words}

Gremlin1 • Mesenchymal stromal cells • Epithelial mesenchymal transition • Human esophageal squamous carcinoma.

\begin{abstract}
Backgroud/Aims: Mesenchymal stromal cells (MSCs) are a major component of the tumor microenvironment (TME). Several studies focusing on tumor-derived MSCs have demonstrated that they exhibit a strong ability to promote the tumor epithelial-mesenchymal transition (EMT). However, the factors mediating these effects are poorly understood. Methods: Quantitative reverse transcription-polymerase chain reaction (qRT-PCR) and immunohistochemistry assays were used to detect the expression of Gremlin1 (GREM1) in human esophageal squamous cell carcinoma (ESCC) tissues. ShRNA silencing, flow cytometry, cell counting kit (CCK8) assay, invasion assay, western blot were used to detect the effect of GREM1 in ECa109, TE-1 cell lines and xenograft tumor models. Results: In the current study, we found that the GREM1 was overexpressed in human ESCC tissues. The conditioned medium from mesenchymal stromal cells (MSCs-CM) enhanced the malignancy of xenograft esophageal tumors in vivo, as well as the cell proliferation, viability and invasion of the esophageal carcinoma cell lines ECa109 and TE-1 in vitro. Furthermore, the shRNA silencing of GREM1 in MSCs (shGREM1-MSCs) reversed the increased malignancy of the esophageal tumor in vivo, while the conditioned medium from shGREM1-MSCs (shGREM1-MSCs-CM) affected the cell cycle and cell invasion in vitro. These processes were accompanied by the EMT in the ECa109 and TE-1 cell lines with an alteration in the expression levels of mesenchymal and epithelial markers. Furthermore, D. Hong, T. Liu andW. Huang contributed equally to this work.

Qiuling Xiang and Xinchun Zhang

Center for Stem Cell Biology and Tissue Engineering, Sun Yat-Sen University

No.74, Zhongshan 2nd Road, Yuexiu District, Guangzhou 510080, Guangdong (China)

Tel. +8620-87335822, E-Mail xiangql@mail.sysu.edu.cn, zhxinch@mail.sysu.edu.cn
\end{abstract}


the TGF- $\beta /$ BMP (transforming growth factor-beta/bone morphogenetic protein) signaling pathway participated in the shGREM1-MSCs-CM-induced anti-tumor effect on enhanced esophageal malignancy induced by MSCs-CM treatment. Conclusions: Taken together, our study suggested that GREM1 delivered by MSCs promoted EMT in ESCC in vitro and in vivo, which is partly through TGF- $\beta$ /BMP signaling pathway. The results provide experimental evidence to a potential therapeutic target in the treatment of esophageal cancer.

(C) 2018 The Author(s)

Published by S. Karger AG, Basel

\section{Introduction}

Esophageal squamous-cell carcinoma (ESCC) is one of the two main subtypes of esophageal cancer, which is the sixth most common cause of cancer-related mortality in the world [1]. The incidence of ESCC has risen rapidly in recent years, and more than 450, 000 people have been diagnosed [2]. Tumor metastasis is one of the main reasons causing the high mortality in ESCC patients [3]. The epithelial-mesenchymal transition (EMT), is a process in which epithelial cells lose their cell polarity and cell-cell adhesion ability, and acquire migratory and invasive properties to gain the mesenchymal phenotype. It is an important step leading to tumor metastasis. Decreased expression of the epithelial marker E-Cadherin and an increased profile of distinct mesenchymal markers, such as N-Cadherin, Vimentin, Snail and Twist are the major hallmarks of the EMT phenomena [4]. Features of EMT have been associated with poor prognosis and chemo-resistance in the treatment of cancer [5]. Therefore, the elucidation of mechanisms of EMT regulation could facilitate future efforts to better understand occurrence, recurrence, and metastasis in cancer.

The tumor microenvironment (TME) plays a critical role in orchestrating the growth of tumors [6] and can be classified into two groups: kinds of cells and other components [7]. The kinds of cells are comprised of proliferating tumor cells, fibroblasts, epithelial cells, inherent or specific immune cells, mesenchymal stromal cells (MSCs), and a variety of associated tissue cells, all of which are components of the overall tumor stroma. Other components include cytokines, growth factors, the extracellular matrix, and hormones. The microenvironment is created by and at all times shaped and dominated by the tumor in response to various stimuli. The tumor avoids apoptosis by regulating the expression of proteins, growth factors, and inflammatory mediators and by creating an inflammatory microenvironment to promote its survival and development [8]. Bone marrow derived MSCs are a type of stromal cell with the capacity of self-renewal and differentiation into mesodermal cell lineages [9]. Some studies have shown that MSCs can inhibit the proliferation of tumor cells in vitro [5]. In contrast, other studies suggest that human MSCs may play a role in carcinogenesis and positively modulating tumor growth [10], due to their ability to incorporate within the tumor stroma.

Gremlin1 (GREM1) is an extracellular bone morphogenetic protein (BMP)-specific antagonist [11]. It contains a cysteine knot, as do other extracellular BMP antagonists. The role of GREM1 as a BMP antagonist has been identified in limb development and in kidney and lung branching morphogenesis [5]. Recently, its clinical significance was reported in a variety of human cancers. GREM1 is overexpressed in various human tumors, including sarcoma and carcinomas of the lung, ovary, kidney, breast, colon, and pancreas [12]. Furthermore, MSCs in cancer tissues have been shown to secrete GREM1 to maintain glioblastoma tumor proliferation and hierarchy. In a previous study, Li, et al. [13], reported that the GREM1mediated decrease in bone morphogenetic protein signaling promotes aristolochic acidinduced EMT in HK-2 cells. All of these observations suggest that GREM1 plays a critical role in the progression of EMT.

In this study, we planned to determined the effect of GREM1 silencing in MSCs on esophageal cancer both in vitro and in vivo. In addition, the expression changes of epithelial and mesenchymal markers in ECa109 and TE-1 cells were observed, indicating the occurrence of EMT when cultured in conditioned medium from the scrambled MSCs (scrambled-MSCs-CM) and initiation of reverse EMT after treatment with conditioned medium from GREM1-silenced MSCs (shGREM1-MSCs-CM). Furthermore, BMP and BMP- 


\section{Cellular Physiology Cell Physiol Biochem 2018;47:1785-1799 \begin{tabular}{l|l} 
and Biochemistry Published 10.1159/000491060 & $\begin{array}{l}\text { (c) 2018 The Author(s). Published by S. Karger AG, Basel } \\
\text { www.karger.com/cpb }\end{array}$ \\
\hline
\end{tabular}}

Hong et al.: Gremlin1 Induced by MSCs Promoted EMT in ESCC

related TGF- $\beta$ (transforming growth factor-beta) signal pathway were planned to detect during the process. These results provide evidence to support the conclusion that GREM1 expression is critical to the carcinoma cell transition from the epithelial to mesenchymal states in esophageal cancer cells.

\section{Materials and Methods}

Ethical statement

This study was approved by the Ethics Committee of Sun Yat-Sen University, China.

\section{Cell culture and tissue preparation}

The human esophageal cancer cell lines ECa109 and TE-1 were purchased from the Institute of Biochemistry and Cell Biology (Shanghai, China). The cells were cultured in RPMI1640 supplemented with $10 \%$ fetal bovine serum (FBS; Life Technologies, USA), $100 \mathrm{U} / \mathrm{ml}$ penicillin and $100 \mathrm{mg} / \mathrm{ml}$ streptomycin at $37^{\circ} \mathrm{C}$ with $5 \% \mathrm{CO}_{2}$. MSCs were cultured using a previously described method ${ }^{5}$. Briefly, bone marrow was harvested from the femora and was plated into a culture dished at a concentration of $10^{6}$ cells $/ \mathrm{ml}$ in Iscove's modified Dulbecco's medium (IMDM; HyClone, USA) that was supplemented with 10\% FBS (Life Technologies, USA) and $1 \%$ penicillin/streptomycin at $37^{\circ} \mathrm{C}$ and $5 \% \mathrm{CO}_{2}$. The medium was replaced every 3 days. MSCs at passages 3-5 were used for all experiments. Fourteen pairs of normal human esophageal tissues and human esophageal tumor tissues were provided by Sun Yat-Sen Memorial Hospital.

\section{Quantitative reverse transcription-polymerase chain reaction ( $q R T-P C R$ )}

Total RNA extraction was performed using TRIzol reagent (Life Technologies, USA) according to the manufacturer's instruction. Two microgram of total RNA extracted from the cells was subjected to reverse transcription. The cDNA synthesis was performed with a one-step RT-PCR kit from Takara (Takara, Japan). SYBR Green (Toyobo, Japan) RT-PCR amplification and real time fluorescence detection were performed using the ABI 7300 real-time PCR thermal cycle instrument (ABI, USA), according to the supplied protocol. Relative gene expression was calculated with the $2^{-\Delta \Delta \mathrm{Ct}}$ method. The primer sequences are shown in the Table 1.

\section{Immunohistochemistry}

The sections of normal human esophageal and tumor tissues were washed with PBS and blocked for 60 min in $0.3 \%$ Triton X-100 in phosphate-buffered saline with $5 \%$ bovine serum albumin. The normal human esophageal and human tumor sections were incubated at $4^{\circ} \mathrm{C}$ with anti-GREM1 antibody (Cat. No. ab22138, Abcam, USA). The xenograft tumor sections were incubated overnight with in anti-CD31 (Cat. No. ab28364, Abcam, USA), antiE-cadherin (Cat. No. ab15148, Abcam, USA), anti- $\beta$-catenin (Cat. No. sc-7199, Santa Cruz Biotechnology, USA), anti-vimentin (Cat. No. sc-6260, Santa Cruz Biotechnology, USA) or anti-N-cadherin antibodies (Cat. No. sc-53488, Santa Cruz Biotechnology, USA). HRP-conjugated secondary antibody (Cat. No. ab6759, Abcam, USA) was applied to the slides, and incubated for 1 hour at room temperature. Finally, DAB $/ \mathrm{H}_{2} \mathrm{O}_{2}$ was added to the surface of the slide to develop the color. The slides were visualized with a Nikon ECLIPSE 90i (Nikon, Japan).

\section{Immunofluorescence assays}

Immunofluorescence assays were performed to detect the expression of CD105 and GREM1 in normal and esophageal carcinoma tissues. Firstly, the tissue specimens were fixed in $10 \%$ neutral buffered formalin, ethanol dehydrated, xylene cleared,
Table 1. The primers sequences used for quantitative reverse transcriptionpolymerase chain reaction (qRT-PCR)

\begin{tabular}{ll}
\hline Gene name & \multicolumn{1}{c}{ primers sequences (5' ${ }^{\prime}$ ) } \\
\hline GREM1 & Forward: CACTACCATGATGGTCACAC \\
& Reverse: CTCTCTAGCAGAGACTGTGT \\
E-cadherin & Forward: ACAGCCCCGCCTTATGATT \\
& Reverse: TCGGAACCGCTTCCTTCA \\
N-cadherin & Forward: GAAAGCGGCTGATACTGACC \\
& Reverse: CGTACATGTCAGCCGCTTC \\
3-catenin & Forward: ACTGCTGGGACTCTG \\
& Reverse: TGATGGCGTAGAACAG \\
Vimentin & Forward: TGTCCAAATCGATGTGGATGTTTC \\
& Reverse: TTGTACCATTCTTCTGCCTCCTG \\
Slug & Forward: TTCGGAACACATTACCT \\
& Reverse: TGACCTGTCTGCAAATGCTC \\
Twist & Forward: TGAGCAAGATTCAGACCCTCA \\
& Reverse: ATCCTCCAGACCGAGAAGG \\
GAPDH & Forward: GGTATCGTGGAAGGACTCATGAC \\
& Reverse: ATGCCAGTGAGCTTCCCGT TCAGC \\
\hline
\end{tabular}




\section{Cellular Physiology Cell Physiol Biochem 2018;47:1785-1799 \begin{tabular}{l|l} 
and Biochemistry Published online: June 28, 2018 & $\begin{array}{l}\text { D) } 2018 \text { The Author(s). Published by S. Karger AG, Basel } \\
\text { www.karger.com/cpb }\end{array}$ \\
\hline
\end{tabular}}

Hong et al.: Gremlin1 Induced by MSCs Promoted EMT in ESCC

paraffin embedded, and subsequently dewaxed. The thickness of the paraffin-embedded tissues were between 3 and 5 microns. Double-immunofluorescence staining of CD105 (Cat. no. ab69772, Abcam, USA) and GREM1 (Cat. no. MBS9215630, MyBioSource, USA) was performed using antibodies and they were labeled with green and red fluorophores, respectively, while the nuclei were counterstained with DAPI (blue) and the cells were observed by microscopy.

\section{GREM1 knockdown by RNA Interference}

We used the following shRNA sequences designed against the GREM1: shRNA-1 (sense) 5'-TGCATCGATTTGGATTAAGCCCTTCCTGTCAGGCTTAATCCAAATCGATGTTTTTTC-3', (antisense) 5'-TCGAGAAAAAACATCGATTTGGATTAAGCCTGACAGGAAGGGCTTAATCCAAATCGATGCA-3'; shRNA-2 (sense) 5'ATAACAGAAGCGGTTGATGAT-3', (antisense) 5'-ATCATCAACCGCTTCTGTTAT-3'. Short hairpin RNA (shRNA) targeting Gremlin1 and a scrambled sequence serving as the control were cloned into a lentiviral vector with two other plasmids coding for the lentiviral envelope (pCMV-VSV-G) and packaging proteins (pCMV delta R8.2). These constructs were co-transfected into 293T cells using Lipofectamine 2000 (Life Technologies, USA) and viral particles were collected 48 hours later, as described previously ${ }^{5}$. MSCs were infected with lentivirus containing GREM1 shRNA or the scrambled sequence and the resulting cells were designated as shGREM1-MSCs and scrambled-MSCs, respectively. qPCR was used for the detection of GREM1 mRNA expression.

\section{Flow cytometric MSCs characterization}

The surface expression of MSCs markers was determined via flow cytometry. The MSCs suspensions were incubated with Fluorescein conjugated antibodies for $60 \mathrm{~min}$ at room temperature, including CD29 (Cat. No. BZ181097), CD31 (Cat. No. 351-011), CD44 (Cat. No. BV825662), CD45 (Cat. No. 345808), CD73 (Cat. No. AY261195) and CD90 (Cat. No. CM289877) (All purchase from R\&D Systems, USA). Following incubation, the cells were re-suspended in PBS containing 2\% FBS. A cell sorting analysis was performed using a FACS Caliber Flow Cytometer (BD Biosciences, USA), and the data were analyzed using the Cell Quest-Pro software (BD Biosciences, USA).

\section{Preparation of concentrated conditioned medium (CM)}

After MSCs had reached greater than $80 \%$ confluence, the medium was aspirated, and the cells were rinsed 3 times with PBS. They were then cultured with serum-free DMEM for 24 hours. The medium from $10^{6}$ cells yielded $6 \mathrm{ml}$ of primary CM, which was further de-salted and concentrated 20-fold by centrifugation (4, $000 \times \mathrm{g}$ for $30 \mathrm{~min}$ at $4^{\circ} \mathrm{C}$ ) using ultrafiltration units with a 3-kDa molecular weight cut-off (Amicon Ultra-PL 3; Millipore, Billerica, MA, USA), ultimately yielding $300 \mu \mathrm{l}$ of concentrated CM. Similar to previous reports [14], we used serum-free DMEM (de-salted and concentrated 20-fold) as the vehicle control.

\section{Adipogenic and osteogenic differentiation of MSCs}

MSCs were harvested by $0.25 \%$ trypsin treatment. Cells were seeded at a density of $15 \times 10^{3}$ cells/ $\mathrm{cm}^{2}$ in 24-well plates. The cultures were incubated in either adipogenic or osteogenic differentiation medium for 21 days. The adipogenic differentiation medium was composed of high glucose DMEM (Life Technologies, USA) that was supplemented with $10 \% \mathrm{FBS}, 1 \%$ penicillin/streptomycin, $10 \mu \mathrm{g} / \mathrm{mL}$ insulin (Sigma-Aldrich), $0.5 \mathrm{mM}$ isobutylmethylxanthine (IBMX, Sigma-Aldrich), $0.1 \mathrm{mM}$ indomethacin (SigmaAldrich), and $1 \mu \mathrm{M}$ dexamethasone (Sigma-Aldrich). The osteogenic differentiation medium was composed of high glucose DMEM that was supplemented with 10\% FBS, 1\% penicillin/streptomycin, $10 \mathrm{ng} / \mathrm{mL}$ Fibroblast Growth Factor 2 (FGF-2, BD Biosciences), $10 \mathrm{mM} \beta$-glycerophosphate (Sigma-Aldrich), and 10 nM dexamethasone. The medium was changed every 3 days. After 21 days of differentiation, cells were fixed in 4\% paraformaldehyde for 20 min at room temperature, and stained with Oil Red 0 for adipogenicdifferentiated MSCs and alizarin Red S for osteogenic-differentiated cells. The images were captured using a Nikon ECLIPSE 90i (Nikon, Japan).

Enzyme-linked immunosorbent assay (ELISA)

The concentrations of GREM1 in shGREM1-MSCs-CM and scrambled-MSCs were obtained with ELISA. The scramble-MSCs and shGREM1-MSCs-CM were placed on 96 well plates. After coating, plates were 


\section{Cellular Physiology Cell Physiol Biochem 2018;47:1785-1799 \begin{tabular}{l|l} 
and Biochemistry Published online:June 28, 2018 & $\begin{array}{l}\text { (c) 2018 The Author(s). Published by S. Karger AG, Basel } \\
\text { www.karger.com/cpb }\end{array}$ \\
\hline
\end{tabular}}

Hong et al.: Gremlin1 Induced by MSCs Promoted EMT in ESCC

sequentially washed with PBST buffer, blocked with $1 \%$ BSA, and incubated for $1 \mathrm{~h}$ at $37^{\circ} \mathrm{C}$. Then, antiGREM1 antibody and HRP-conjugated antibody were sequentially added and incubated for $1 \mathrm{~h}$ at $37^{\circ} \mathrm{C}$. The detection was achieved by adding the chromogenic substrate, 3, 3',5, 5' -Tetramethylbenzidine. Absorbance was measured at $450 \mathrm{~nm}$ with an EnSpire multimode plate reader (Perkin Elmer, Waltham, Massachusetts).

\section{Analysis of cell cycle phase by flow cytometry}

Forty-eight hours after treatment with conditioned medium, the ECa-109 and TE-1 cells were resuspended in PBS twice before fixation by dropwise addition into 95\% precooled ethanol. Prior to analysis, the cells were warmed, centrifuged at $450 \mathrm{~g}$ for $5 \mathrm{~min}$, re-suspended twice in PBS, and then stained with PI (containing RNase A at $50 \mu \mathrm{g} / \mathrm{ml}$ ) at room temperature in the dark for $30 \mathrm{~min}$. The DNA content was analyzed with flow cytometry using the CellQuest program (Becton-Dickinson and Co., USA)._ENREF_25

\section{Cell counting kit (CCK8) assay}

In order to detect proliferation, cell suspensions were plated into 96 -well plates ( $5 \times 10^{4}$ cells $/$ well), 10 $\mu \mathrm{l}$ CCK Solution was added to the wells and the cells were incubated for $2.5 \mathrm{~h}$ in the incubator. The MultiVolume Spectrophotometer System (BioTek Epoch, USA) was used to measure the absorbance at $450 \mathrm{~nm}$. The results were calculated as [OD value of Sample - OD value of blank]/[OD value of blank] \%. All data were collected from three independent experiments.

\section{Invasion assay}

The invasion assay was performed using BD Biocoat matrigel invasion chambers. In brief, $5 \times 10^{4}$ cells were seeded on top of a matrigel-coated transwell in $100 \mu \mathrm{L}$ of serum-free medium. The transwells were embedded into the conditioned medium, and cells were allowed to invade for $48 \mathrm{~h}$. At the end of the assay, invading cells on the bottom side of the well were stained with Giemsa, photographed, and counted under a microscope. This experiment was independently repeated in triplicate.

\section{Western blot analysis}

The proteins were extracted from the cells using $100 \mu \mathrm{l}$ Lami Buffer/(35 mm well), separated electrophoretically on a 10\% SDS-polyacrylamide gel and transferred onto polyvinylidene fluoride membranes. After blocking with 5\% skim milk, the membrane was incubated with antibodies to GREM1 (1:500) (Cat. No. ab57139; Abcam, USA), TGF- $\beta$ (Cat. No. 3711; Cell Signaling Technology, USA), BMP-4 (Cat. No. 4680; Cell Signaling Technology, USA), p-Smad2/3 (Cat. No. ab63399; Abcam, USA), or p-Smad1/5/8 (Cat. No. NB100-56443, Novus Biologicals, Inc., USA) (all 1:1000) at $4{ }^{\circ} \mathrm{C}$ and was incubated overnight. The membrane was then washed three times with TBST for 5 minutes each with the corresponding diluted secondary antibody (HRP-conjugated secondary antibody, 1: 1000, Forevergen, China) and incubated at room temperature for 1-2 hours. After washing with TBST three times for 10 minutes each, the signals were detected by enhanced chemiluminescence (ECL, Forevergen, China). GAPDH (1:2000) was used as an internal reference for comparison.

\section{Xenograft tumor models}

The inoculation area on the mice was cleaned and sterilized with ethanol and iodine solutions. ECa109 or TE- 1 cells $\left(2 \times 10^{6}\right.$ cells in $200 \mu$ PBS $)$ with or without the scrambled-MSCs or shGREM1-MSCs $\left(2 \times 10^{6}\right.$ cells in $200 \mu \mathrm{l}$ PBS) were subcutaneously injected into the unilateral flanks of 6-week-old BALB/c nude mice. Tumors were observed 4 weeks after injection. The implanted mice were observed daily. Tumor volume $\left(\mathrm{mm}^{3}\right)$ was calculated using the following equation: Tumor volume $=\left(\mathrm{a}^{2} \times \mathrm{b}\right) / 2$, where "a" is the width of the tumor (small diameter) and "b" is the length (large diameter) (mm).

\section{ENREF_26_ENREF_27 Statistical analysis}

Data are presented as mean \pm SEM and were analyzed with Graphpad Prism 6 software. Unpaired Student's t-tests and One-way ANOVA were used to determine significant differences. A p value less than 0.05 was considered to be significantly different. 


\section{Cellular Physiology Cell Physiol Biochem 2018;47:1785-1799 \begin{tabular}{l|l} 
and Biochemistry & DOI: 10.1159/000491060 \\
Published online: June 28, 2018 & $\begin{array}{l}\text { C } 2018 \text { The Author(s). Published by S. Karger AG, Basel } \\
\text { www.karger.com/cpb }\end{array}$
\end{tabular} \\ Hong et al.: Gremlin1 Induced by MSCs Promoted EMT in ESCC}

\section{Results}

\section{High expression of GREM1 and CD105 in esophageal carcinoma tissues}

The level of GREM1 between normal esophageal and carcinoma tissues were determined. The average level of GREM1 mRNA in esophageal carcinoma was about 2.5 times that of the normal tissues $(\mathrm{p}<0.05$, Fig. $1 \mathrm{~A})$. The difference was confirmed by the immunohistochemical analysis, which showed that GREM1 expression was significantly higher in the esophageal carcinoma than in the normal tissue $(\mathrm{p}<0.05$, Fig. $1 \mathrm{~B}-\mathrm{C})$. These results indicate that there were higher expression levels of GREM1 in esophageal tissues compared with normal esophageal tissues. The expressions of the MSCs marker CD105, and GREM1 were detected by double color immunofluorescence assay. The result of immunofluorescence assay revealed that CD105 and GREM1 were highly expressed in esophageal carcinoma tissues (Fig. 1D). The expressions of CD105 and GREM1 double-positive cells in esophageal carcinoma were much greater than that of normal tissue.

\section{Characteristic of MSCs after GREM1 downregulation}

The specific cell surface antigens on the MSCs were detected by flow cytometry. The scrambled-MSCs strongly expressed CD29 (99.6\%), CD44 (95.1\%), CD73 (98.0\%) and CD90 (98.6\%), but not CD31 (0.607\%) or CD45 (4.19\%) (Fig. 2A, upper). After treatment with the short hairpin RNA targeting Gremlin1 (shGREM1), the MSCs failed to show any significant change in the expressions of different cell surface antigens (CD29, 99.7\%; CD31, 0.314\%; CD44, 96.5\%; CD45, 0.566\%; CD73, 99.4\%; and CD90, 100\%) (Fig. 2A, lower). Next, we found that GREM1 silencing did not decreased the capacity of adipogenic and osteogenic differentiation. In other words, the pluripotency for osteogenesis and adipogenesis was still shown in the MSCs after GREM1 silencing (Fig. 2B). A CCK8 assay was performed to detect the expansion of control and shGREM1 MSCs, but the resulting GREM1 silencing in MSCs had no effect on cell proliferation (Fig. 2C). In addition, the efficiency of shRNA-1 and shRNA-2 in MSCs GREM1 expression was evaluated by qPCR, showing that the expression of GREM1

Fig. 1. The differential expression of GREM1 in normal esophageal and carcinoma tissues. (A) The GREM1 mRNA expression level was significantly higher in esophageal carcinoma tissues than that in the normal tissues, as determined by qPCR. ${ }^{*} \mathrm{p}<0.05$ vs Normal. $\mathrm{n}=14$. (B) Immunostaining data showed the higher expression level of GREM1 in carcinoma compared to the normal tissue. Scale Bar $=50$ $\mu \mathrm{m}$. (C) Quantified data of GREM1 positive cells. ${ }^{*} \mathrm{p}<0.05$ vs Normal. $\mathrm{n}=14$. (D) In a double color immunofluorescence assay, highly positive signals were detected for CD105 and GREM1 in esophageal carcinoma tissues compared to the normal tissues. The cell nuclei were stained blue. Scale Bar= 200 $\mu \mathrm{m}$.

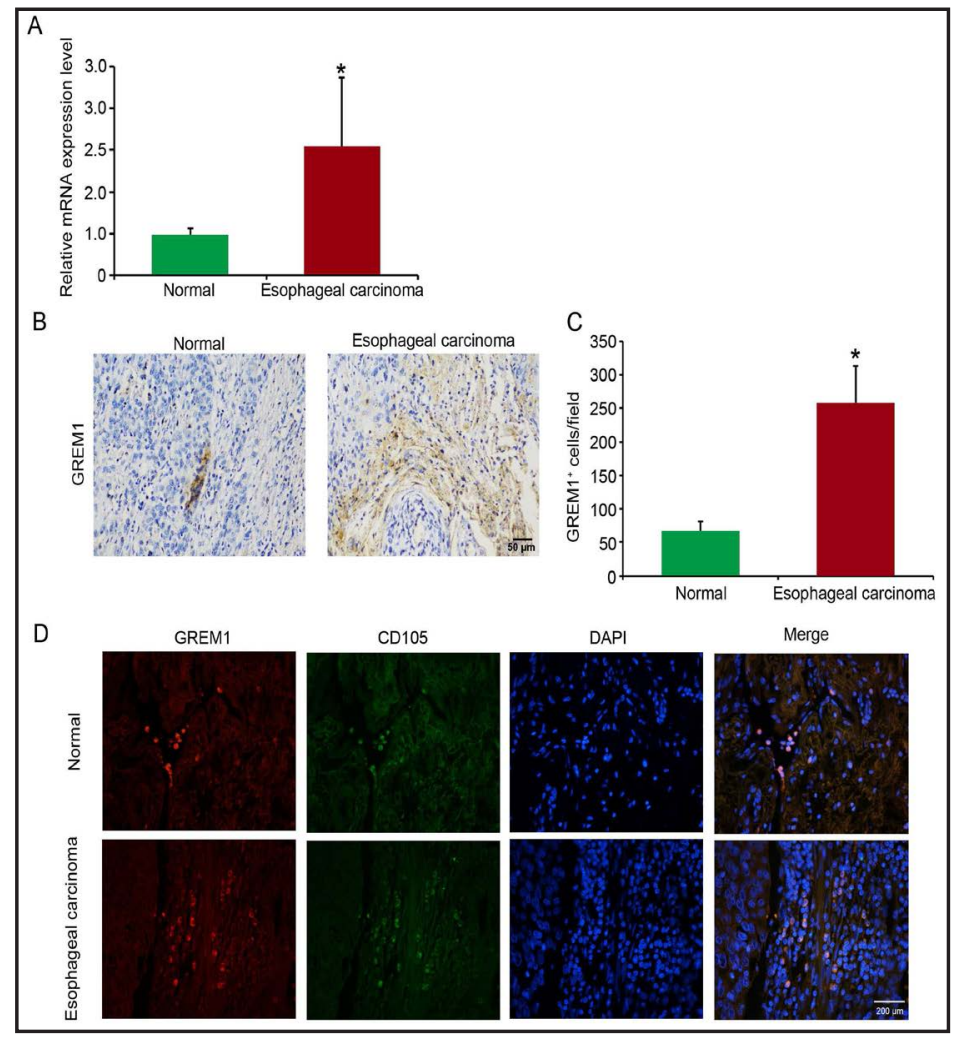


Fig. 2. ShRNA-GREM1 MSCs were constructed. (A) Detection of the specific cell surface antigens (CD29, CD31, CD44, CD45, CD73 and CD90) on scrambled-MSCs and shGREM1-MSCs by flow cytometry. (B) Adipogenic and osteogenic differentiation of MSCs. After adipogenic and osteogenic differentiation, cells were stained with Oil Red 0 for adipogenicdifferentiated MSCs and alizarin Red S for osteogenic-differentiated cells. (C) After transfection with shRNA-1, the cell proliferation of scrambled-MSCs and shGREM1MSCs was determined by CCK8 assay. (D) The efficacy of shRNA-1 and shRNA-2 in GREM1 silencing was evaluated by qPCR. The expression of GREM1 mRNA decreased significantly by about $80 \%$ and $50 \%$ in MSCs after transfection with shRNA-1 and shRNA-2, respectively. $\quad{ }^{*} \mathrm{p}<0.05$ vs the scrambled-MSCs group. (E) The GREM1 concentration in shGREM1-MSCs-CM was determined with ELISA. The GREM1 concentration decreased significantly in shGREM1-MSCs-

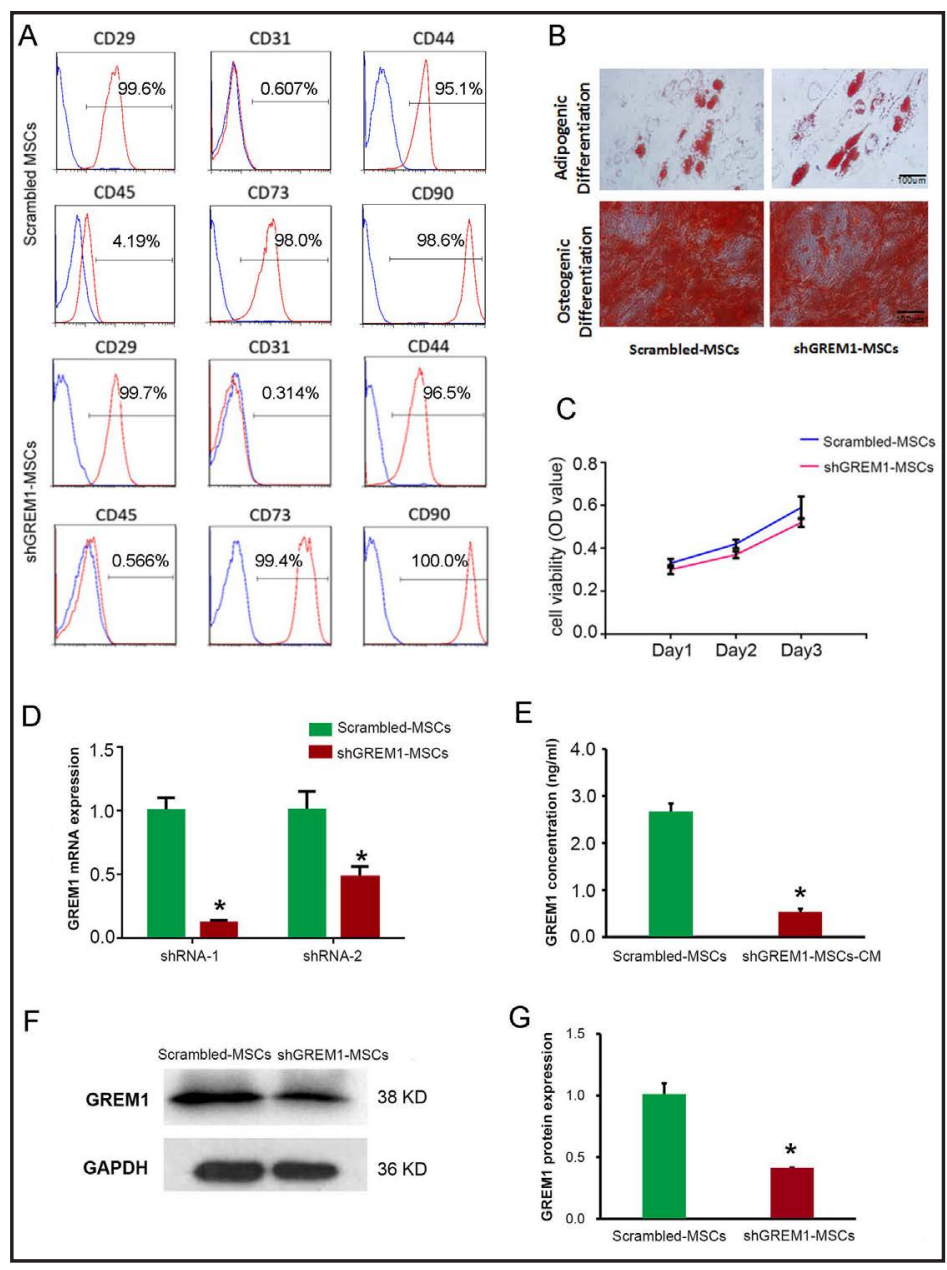
CM. ${ }^{*}$ p $<0.05$ vs the scrambled-MSCs group. (F) The GREM1 protein expression level decreased significantly in the shGREM1-MSCs, as determined by western blotting. (G) The quantified GREM1 protein expression data was analyzed by Image J software. ${ }^{*} \mathrm{p}<0.05$ vs the scrambled-MSCs group.

mRNA was significantly decreased about $80 \%$ and 50\% after transfected with shRNA-1 and shRNA-2, respectively (Fig. 2D). Therefore, shRNA-1 was chosen for the following experiments. The GREM1 protein was detected by ELISA and western blotting after shRNA-1 transfection. We found that the concentration and protein level of GREM1 decreased about $75.65 \%$ and $59.4 \%$ in the shGREM1-MSCs-CM group, respectively, when compared with the scramble-MSCs group ( $\mathrm{p}<0.05$, Fig. 2E-G). Collectively, these data confirmed the efficiency of GREM1 silencing. And treated with shGREM1, MSCs maintained their characteristic, the capacity of adipogenic and osteogenic differentiation.

\section{ShGREM1-MSCs-CM reversed EMT in ECa109 and TE-1 cells}

A typical morphological change was observed in ECa109 and TE-1 cells with the scrambled-MSCs-CM and shGREM1-MSCs-CM treatment (Fig. 3A). Compared to the untreated cells, the ECa109 and TE-1 cells treated with scrambled-MSCs-CM morphologically changed from a polygonal to a bipolar shape, and extended more antennae. However, the application of shGREM1-MSCs-CM reversed this morphological alteration in ECa109 and TE-1 cells. Simultaneously, treatment with scrambled-MSCs-CM decreased the E-cadherin mRNA level. In the shGREM1-MSCs-CM group, the decrease was partly abrogated. We also found that the application of scrambled-MSCs-CM increased the mRNA levels of N-cadherin, beta-catenin, 
Fig. 3. ShGREM1-MSCs-CM altered the EMT in ECa109 and TE-1 cells. (A) Typical morphological change in Eca109 or TE-1 cells after the treatment with scrambled-MSCs-CM or shGREM1-MSCsCM. Compared to the control cells, the ECa109 and TE- 1 cells treated with scrambled-MSCs-CM morphologically changed from a polygonal to a bipolar shape. However, the morphological change in shGREM1-MSCs-CM group was obviously reversed both in ECa109 and TE-1 cells. Scale Bar=100 $\mu \mathrm{m}$. (B) Incubation with scrambledMSCs-CM reduced the E-cadherin mRNA level and increased the expressions of $\beta$-catenin, vimentin, $\mathrm{N}$-cadherin, Slug, and Twist compared to the control group, while the shGREM1-MSCs-CM led to an increase in the E-cadherin mRNA level and a decrease in the $\beta$-catenin, vimentin, $\mathrm{N}$-cadherin, Slug, and Twist mRNA levels compared to those

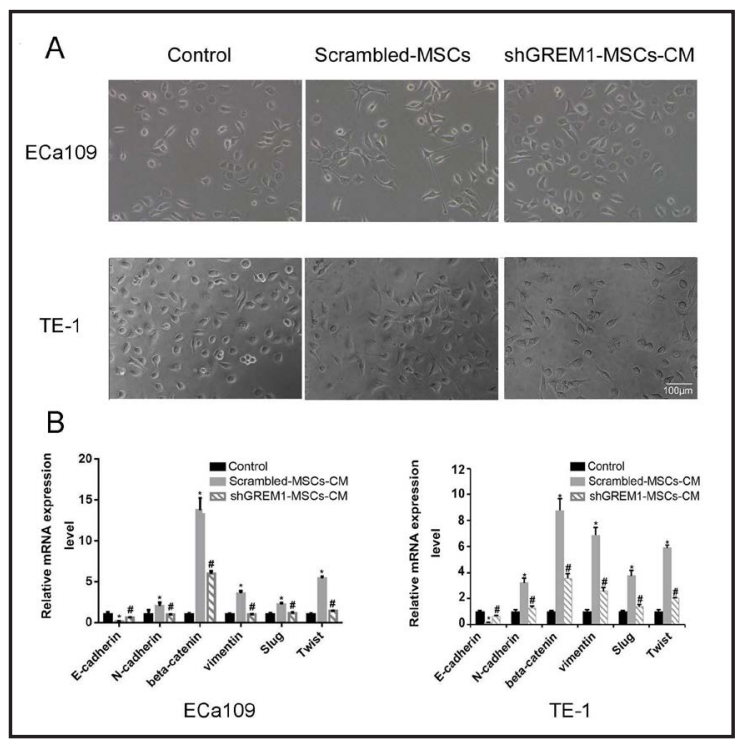
of ECa109 cells treated with scrambled-MSCs-CM.

In TE-1 cells treated with scrambled-MSCs-CM, the mRNA expression level of E-cadherin decreased and those of $\beta$-catenin, vimentin, $\mathrm{N}$-cadherin, Slug, and Twist increased compared to the control group. This effect decreased in the presence of the shGREM1-MSCs-CM, showing the increased mRNA expression level of E-cadherin and the decreased levels of $\beta$-catenin, vimentin, $\mathrm{N}$-cadherin, Slug, and Twist.

Fig. 4. ShGREM1-MSCs-CM altered cell proliferation and viability in ECa109 and TE-1 cells. (A) Flow cytometry analysis showed that cell ratio at $\mathrm{G}_{1}$ phase decreased and increased at $S$ phase in the scrambled-MSCs-CMtreated ECa109 cells, while the ShGREM1-MSCs-CM reversed the process, leading to an increasing cell number at $G_{1}$ phase, and a decreasing ratio at $S$ phase. Quantified cell cycle analysis data in ECa109 cells were shown. (B) In TE-1 cells treated with scrambledMCSs-CM, the cell ratio at G1 phase decreased and increased at $S$ phase. This cell cycle alteration was counteracted in the presence of shGREM1-MSCs-CM, resulting in the increase in ratio of cells at G1 phase and a decreased ration of cells at S phase. Quantified cell

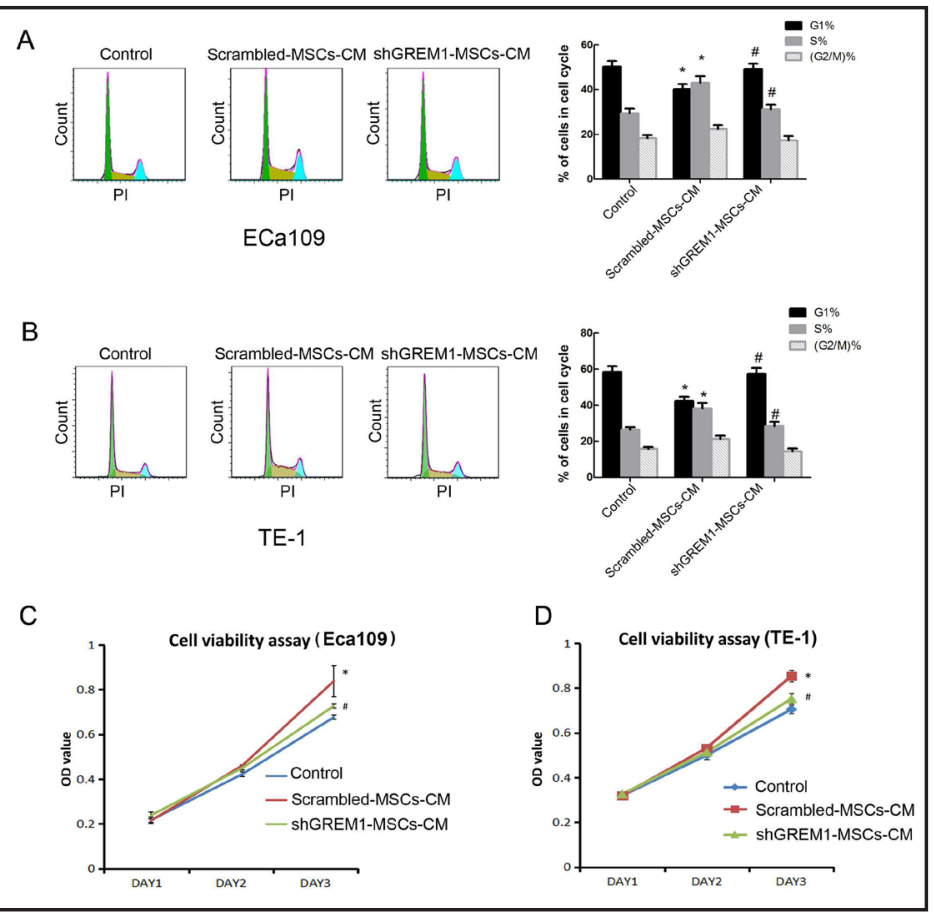
cycle analysis data in TE-1 cells were shown. (C-D) Cell viability was promoted in the scrambled-MSCsCM-treated ECa109 and TE-1 cells, while the application of shGREM1-MSCs-CM reduced the cell viability compared to the scrambled-MSCs-CM-treated cells. ${ }^{*} \mathrm{p}<0.05$ vs the control group, ${ }^{\#} \mathrm{p}<0.05$ vs the scrambledMSCs-CM group.

vimentin, Slug, and Twist, while the shGREM1-MSCs-CM conditioned medium counteracted these increased mRNA levels in ECa109 and TE-1 cells ( $p<0.05$, Fig. 3B). 
ShGREM1-MSCS-CM attenuated ECa109 and TE-1 cell proliferation and viability induced by MSCs-CM

Since the scrambled-MSCs-CM and shGREM1-MSCs-CM affected the EMT capacity of ECa109 and TE-1 cells, we used flow cytometry to determine whether either scrambledMSCs-CM or shGREM1-MSCs-CM could alter the cell cycle. In the presence of the scrambleMSCs-CM, the ratio of ECa109 cells at the G1 phase decreased, while the ratio of cells at S phase increased compared to untreated cells. However, treatment with shGREM1-MSCs-CM reversed this cell cycle alteration, leading to a similar pattern as the untreated cells $(\mathrm{p}<0.05$, Fig 4A). Comparable data was obtained in the TE- 1 cells ( $p<0.05$, Fig. 4B). Next, the cell viability was measured with a CCK8 assay. Treatment with scrambled-MSCs-CM significantly enhanced the viability of both ECa109 and TE-1 cells compared to the control group. In contrast, the shGREM1-MSCs-CM attenuated the increase that was induced by scrambledMSCs-CM (p<0.05, Fig. 4C-D).

\section{ShGREM1 inhibited the invasion ability of esophageal cancer cells}

Since the scrambled-MSCs-CM and shGREM1-MSCs-CM altered the proliferative pattern of ECa109 and TE-1cells, we used a transwell assay to analyze whether cell invasion were affected. $48 \mathrm{~h}$ after incubation with scrambled-MSCs-CM, the number of invading ECa109 and

Fig. 5. ShGREM1-MSCs-CM altered the EMT partly by TGF- $\beta$ and BMP4 signal pathway .(A) ScrambledMSCs-CM treatment increased the number of invading cells compared to the control group, while shGREM1-MSCs-CM weakened the cell invasion promoted by the scrambled-MSCs-CM treatment. (B) Application of scrambledMSCs-CM promoted the invasion of TE-1 cells compared to the control group. ShGREM1-MSCs-CM treatment decreased the number of invading cells compared to that of cells treated with scrambledMSCs-CM. (C-D) Western blotting analysis showed that scrambledMSCs-CM increased the expression levels of TGF- $\beta$ and p-samd2/3, and decreased those of BMP 4 and $\mathrm{p}$-samd $1 / 5 / 8$ compared to the untreated ECa109 and TE-1 cells (control). The application of shGREM1-MSCs-CM decreased TGF- $\beta$ and $p$-samd $2 / 3$ expression and increased that of BMP4 and p-samd1/5/8. In the presence of $1 \mu \mathrm{M}$ SB431542, a TGF- $\beta$ inhibitor, the scrambled-MSCs-CM treatment did not alter the expression levels of TGF- $\beta$, p-samd2/3, BMP4 or p-samd1/5/8 compared to the scrambled-MSCs-CM+DMSO group. ${ }^{*} \mathrm{p}<0.05$ vs control group, ${ }^{*} \mathrm{p}<0.05$ vs scrambled-MSCs-CM group, \&p<0.05 vs scrambled-MSCs-CM+DMSO group.

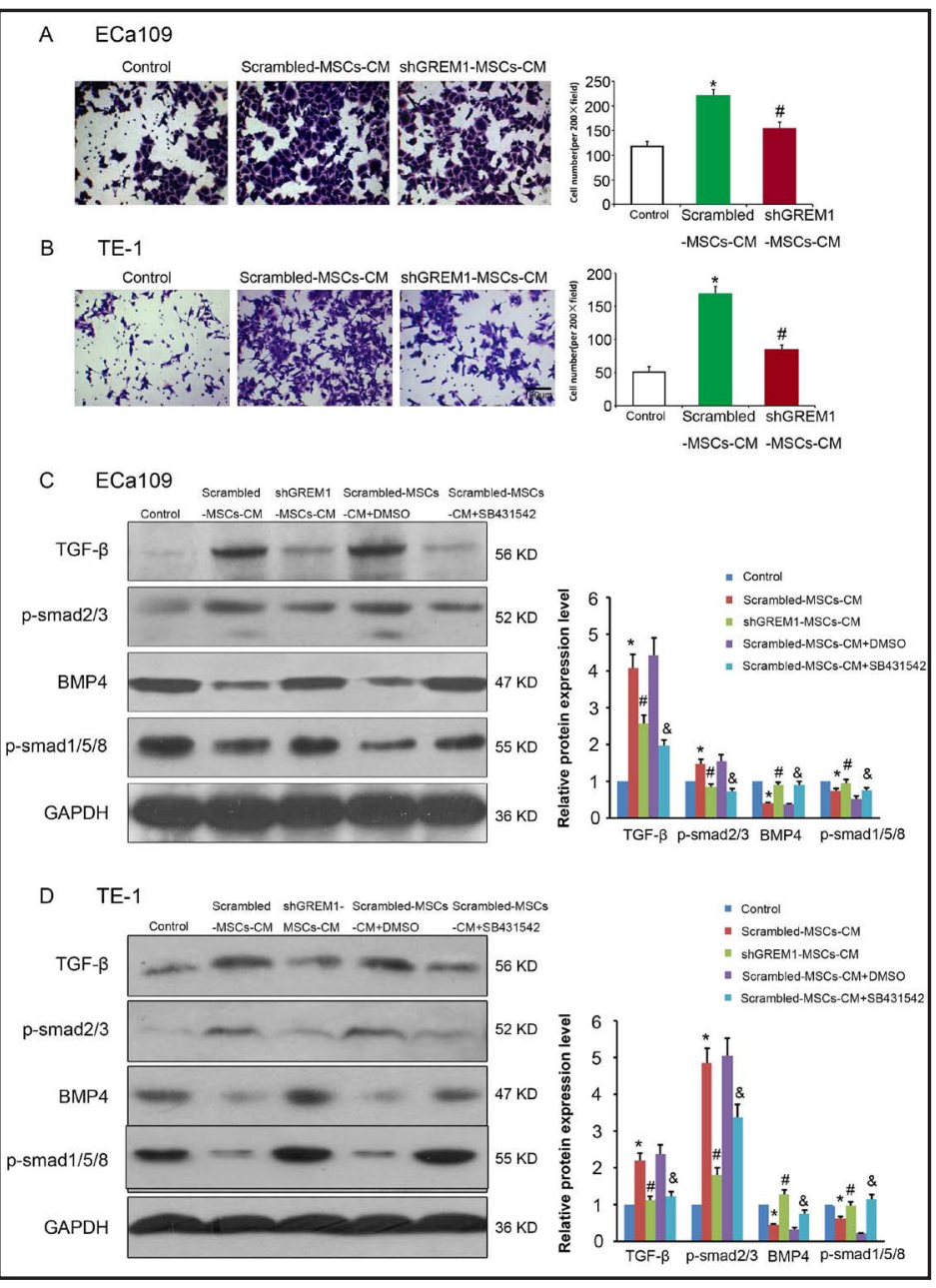


TE- 1 cells $(223 \pm 42$ and $173 \pm 35$, respectively) was significantly increased compared to the control group ( $128 \pm 20$ and $53 \pm 18$, respectively) ( $p<0.05$, Fig. $5 A-B)$, indicating a stronger invasion capacity in the presence of scrambled-MSCs-CM. In contrast, the invasion ability in the shGREM1-MSCs-CM group was obviously decreased as compared to the scrambledMSCs-CM group. The number of invading cells was significantly lower than that in the scrambled-MSCs-CM group ( $\mathrm{p}<0.05$, Fig. 5A-B). This result indicated that the enhanced cell invasion induced by scrambled-MSCs-CM was negatively regulated by GREM1 silencing in esophageal carcinoma cells.

\section{GREM1-induced EMT alteration was mediated by the BMP/TGF- $\beta$ signaling pathway}

Since Gremlin 1 is a BMP antagonist, we determined whether the effect of scrambledMSCs-CM and shGREM1-MSCs-CM were mediated by the BMP/TGF- $\beta$ signaling pathway. The expression level of the TGF- $\beta$ protein increased significantly in the ECa109 cells treated with scrambled-MSCs-CM compared to the control group ( $p<0.05$, Fig. $5 \mathrm{C})$, while the cells incubated in shGREM1-MSCs-CM led to an apparent decrease in TGF- $\beta$ expression compared to the scrambled-MSCs-CM group ( $p<0.05$, Fig. 5C). In the presence of GREM1, the scrambled-

Fig. 6. The effects of TGF- $\beta$ and BMP4 signal pathway to MSCs-CM induced EMT. (A) Flow cytometry analysis showed that cell ratio at $\mathrm{G}_{1}$ phase decreased and increased at $S$ phase in the scrambled-MSCsCM-treated ECa109 cells, while the shGREM1-MSCs-CM reversed the process, leading to an increasing cell number at $G_{1}$ phase, and a decreasing ratio at $S$ phase. In the presence of $1 \mu \mathrm{M}$ SB431542, a TGF- $\beta$ inhibitor, the scrambledMSCs-CM treatment did not alter the cell cycle as compared to the scrambled-MSCs-CM+DMSO group. (B) Similar results were shown in TE-1 cells. Quantified cell cycle analysis data in ECa109 cells (C) and TE-1 cells (D) were shown. (E) Scrambled-MSCs-CM treatment increased the number of invading cells compared to the control group, while shGREM1-MSCs$\mathrm{CM}$ weakened the cell invasion promoted by the scrambledMSCs-CM treatment. SB431542 inhibited the stimulation effect of scrambled-MSCs-CM+DMSO. (F) Similar results were shown in TE-1 cells. Quantified cell invasion data in ECa109 cells (G) and TE-1 cells $(\mathrm{H})$ were shown. ${ }^{*} \mathrm{p}<0.05$ vs control group, ${ }^{*} \mathrm{p}<0.05$ vs scrambled-MSCs-CM group, $\& \mathrm{p}<0.05$ vs scrambled-MSCs$\mathrm{CM}+\mathrm{DMSO}$ group.

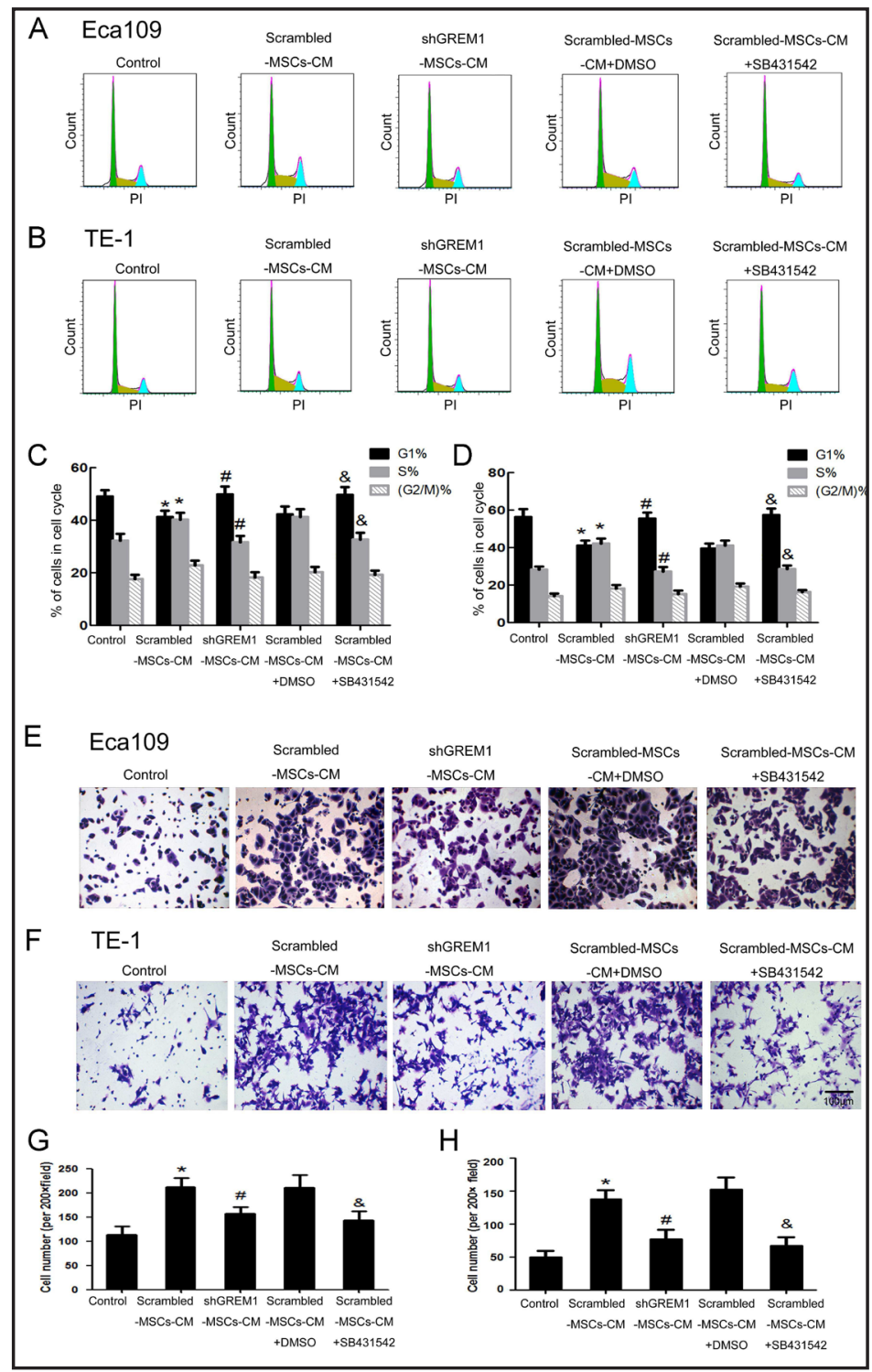


MSCs-CM induced upregulation of TGF- $\beta$ was decreased ( $p<0.05$, Fig. $5 C$ ), while the DMSO control did not show a decrease in the expression of TGF- $\beta$. A similar pattern was observed in the altered expression of the $\mathrm{p}$-samd $2 / 3$ protein. Contrastingly, the level of BMP 4 expression decreased in the presence of scrambled-MSCs-CM ( $p<0.05$, Fig. 5C), and shGREM1-MSCs-CM failed to lead to a drastic decrease in BMP4 expression compared to the control group. The TGF- $\beta$ inhibitor, SB431542, was also shown to counteract the effect of scrambled-MSCs-CM on BMP4 expression. A similar pattern as that of BMP4 was observed in the expression of the $\mathrm{p}$-samd1/5/8 protein ( $\mathrm{p}<0.05$, Fig. $5 \mathrm{C}$ ). We observed the same protein expression change in TGF- $\beta$, p-samd $2 / 3$, BMP4 and p-samd $1 / 5 / 8$ in TE- 1 cells when treated with scrambledMSCs-CM, shGREM1-MSCs-CM and SB431542 ( $\mathrm{p}<0.05$, Fig. 5D). Flow cytometry analysis showed that cell ratio at $G_{1}$ phase decreased and increased at $S$ phase in the scrambledMSCs-CM-treated ECa109 cells, while the shGREM1-MSCs-CM reversed the process, leading to an increasing cell number at $G_{1}$ phase, and a decreasing ratio at $S$ phase. In the presence of SB431542, TGF- $\beta$ inhibitor, the scrambled-MSCs-CM treatment did not alter the cell cycle as compared to the scrambled-MSCs-CM+DMSO group ( $\mathrm{p}<0.05$, Fig. 6A, 6C). Similar results were shown in TE-1 cells (Fig. 6B, 6D). Scrambled-MSCs-CM treatment increased the number of invading cells compared to the control group, while shGREM1-MSCs-CM weakened the cell invasion promoted by the scrambled-MSCs-CM treatment. SB431542 inhibited the stimulation effect of scrambled-MSCs-CM+DMSO (p<0.05, Fig. 6E, 6G). Similar results were shown in TE- 1 cells $(p<0.05$, Fig. $6 \mathrm{~F}, 6 \mathrm{H})$. Collectively, these findings demonstrate that the shGREM1-MSCs-CM-controlled EMT alteration in esophageal carcinoma cells was mediated by the BMP4/TGF- $\beta$ signaling pathway.

GREM1 silencing had an anti-tumor effect on MSCs-induced malignancy in ECa109 and TE-1 xenografts

In the ECa109 xenograft group, the average tumor size reached a volume of $\sim 250 \mathrm{~mm}^{3}$ (Fig. 7A). The average tumor volume in mice injected with ECa109 and scrambled-MSCs had increased to $\sim 620 \mathrm{~mm}^{3}$, but the co-injection of ECa109 and shGREM1-MSCs attenuated the growth of the tumor to $\sim 300 \mathrm{~mm}^{3}$ as compared to the ECa109 and scrambled-MSCs co-injection group ( $p<0.05$, Fig. 7B). Similar results were obtained in the TE- 1 cells. TE- 1 xenograft led to the genesis of tumor with the size of $\sim 1200 \mathrm{~mm}^{3}$ (Fig. 7C). Co-injection of TE1 and scrambled-MSCs increased the tumor size to $\sim 3100 \mathrm{~mm}^{3}$. The tumor growth in coinjection of TE-1 and shGREM1-MSCs group was decreased to $\sim 1500 \mathrm{~mm}^{3}$ as compared to the TE1 and scrambled-MSCs co-injection group ( $p<0.05$, Fig. 7D). Furthermore, ECa109 cells simulated the features of vascular endothelial cells and formed the typical capillary structure in the xenograft model. We found that the average number of capillaries increased significantly to $\sim 12$ per square millimeter in scrambled-MSCs group, while the shGREM1-

Fig. 7. ShGREM1-MSCs inhibited the increasing malignancy of esophageal carcinoma xenografts induced by scrambled-MSCs. (A) The size of the tumors formed by ECa109 cells and scrambledMSCs increased significantly compared to the tumors formed by ECa109 cells only. Co-injection of shGREM1-MSCs and ECa109 decreased the average tumor size compared to the tumors formed by ECa109 cells and scrambled-MSCs. (B) The quantified tumor volume $\left(\mathrm{mm}^{3}\right)$ data of the Eca109 cell line. (C) The size of tumor xenografts from TE-1 cells and scrambled-MSCs increased

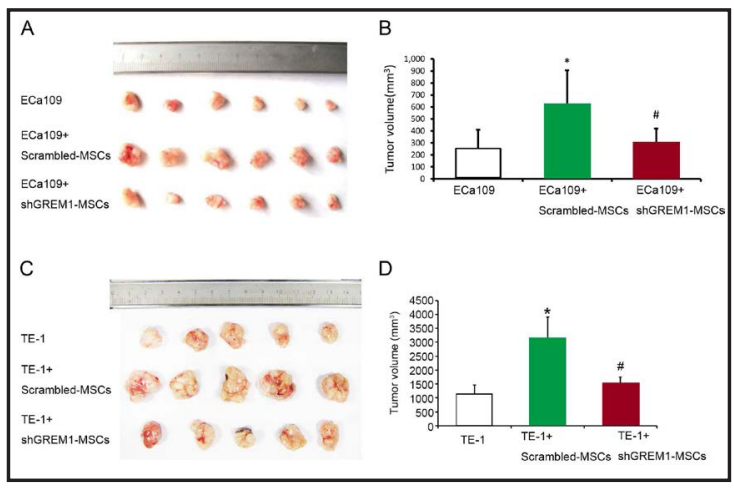
dramatically compared to the xenografts formed by TE-1 cells only, while the shGREM1-MSCs significantly reduced the average tumor size when co-infected with TE-1 cells, in contrast to the tumors formed by TE-1 cells and scrambled-MSCs. (D) The quantified tumor volume $\left(\mathrm{mm}^{3}\right)$ data of TE- 1 cells. ${ }^{*} \mathrm{p}<0.05$ vs the control group, ${ }^{\sharp} \mathrm{p}<0.05$ vs the scrambled-MSCs group. 
Fig. 8. ShGREM1-MSCs altered the EMT in xenograft tumors. (A) The expression of E-cadherin decreased and the expression of $\beta$-catenin, vimentin, and $\mathrm{N}$-cadherin increased in the tumors formed by ECa109 cells and scrambled-MSCs compared to the tumors formed by ECa109 cells only. In contrast, the co-injection of ECa109 cells and shGREM1-MSCs reversed the EMT induced by scrambled-MSCs, leading to the increased expression of E-cadherin and the decreased expression of $\beta$-catenin, vimentin and N-cadherin. Scale Bar $=50 \mu \mathrm{m}$. (B) In tumors formed by TE-1 cells only, the expression level of E-cadherin was higher and the expressions of $\beta$-catenin, vimentin, and $\mathrm{N}$-cadherin were lower than in the tumors formed by TE-1 cells

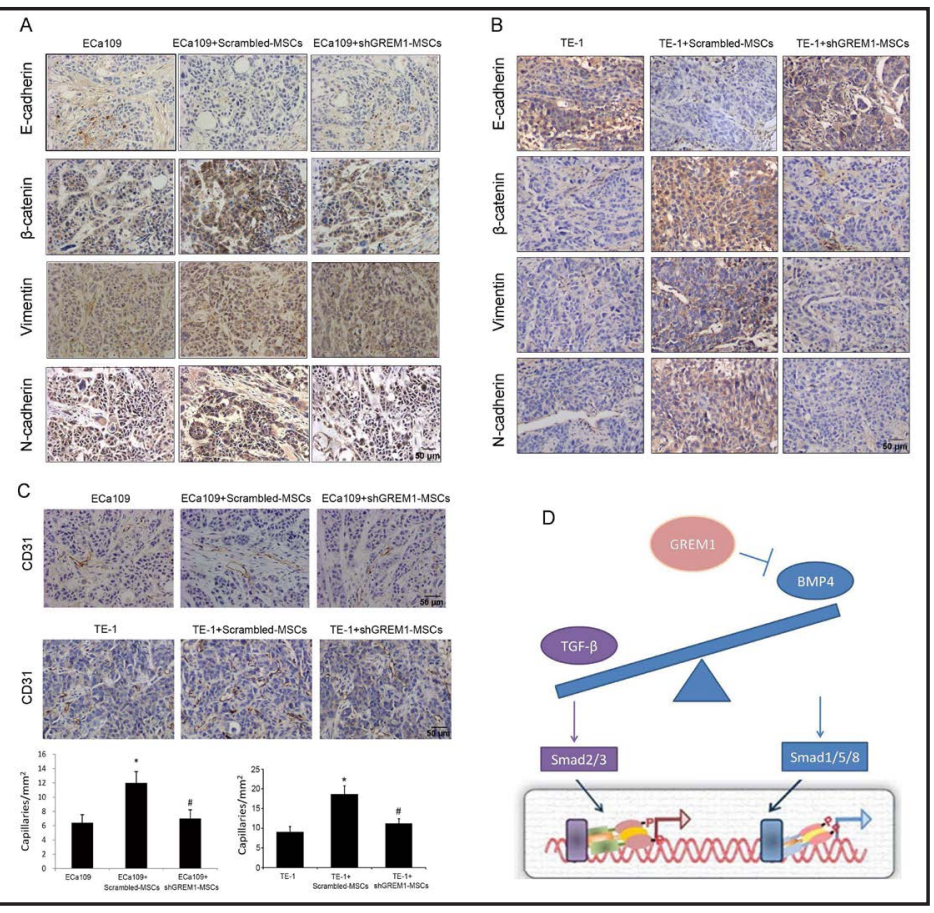
and scrambled-MSCs. Tumors

formed by TE-1 cells and shGREM1-MSCs significantly counteracted this process, showing the increased expression of E-cadherin and decreased expression of $\beta$-catenin, vimentin, and $\mathrm{N}$-cadherin compared to the tumors formed by TE-1 cells and scrambled-MSCs. (C) The expression of CD31 in tumor xenografts. The average number of capillaries increased in tumors formed by ECa109 or TE-1 cells with scrambled-MSCs, while the expression of CD31 decreased when ECa109 or TE-1 cells were co-injected with shGREM1-MSCs, compared to the tumors formed by esophageal carcinoma cells and scrambled-MSCs. Quantified data were also shown. Scale Bar $=50 \mu \mathrm{m} .{ }^{*} \mathrm{p}<0.05$ vs control group, ${ }^{*} \mathrm{p}<0.05$ vs scrambled-MSCs group. (D) A scheme showing the GREM1 regulated the TGF- $\beta$ /BMP signaling pathway during EMT.

MSCs decreased the capillary number to $\sim 6$ ( $p<0.05$, Fig. 8C). A similar tendency was observed in TE- 1 cells. The capillary number increased from $\sim 10$ in TE- 1 xenograft tumors to 18 in tumors in those with scrambled-MSCs. In contrast, only $\sim 11$ capillaries were found in tumors formed by TE- 1 cells and shGREM1-MSCs, which was significantly less than of the scrambled-MSCs group ( $<<0.05$, Fig. 8C).

\section{ShGREM1-MSCs reversed EMT in ECa109 cells in vivo}

The expression levels of the epithelial marker E-cadherin and the mesenchymal markers $\beta$-catenin, vimentin, and $\mathrm{N}$-cadherinin in xenografts were detected by immunohistochemistry. The expression of the epithelial marker E-cadherin decreased, while the expression of the mesenchymal markers $\beta$-catenin, vimentin, and $\mathrm{N}$-cadherin increased in the tumors injected with either scrambled-MSCs ECa109 or TE-1. Nevertheless, the co-injection of shGREM1MSCs with tumors increased the expression of E-cadherin but decreased the expressions of $\beta$-catenin, vimentin and N-cadherin (Fig. 8A-B). This indicate that shGREM1-MSCs inhibited the EMT that was initiated by the scrambled-MSCs in Eca109 and TE-1 cells. Simulated diagram showed GREM1 regulated BMP/TGF- $\beta$ signaling pathway during EMT (Fig. 8D).

\section{Discussion}

In this study, we explored the role of GREM1 in the EMT of esophageal cancer both in vivo and in vitro. Firstly, we found that GREM1 was more highly expressed in esophageal 


\section{Cellular Physiology Cell Physiol Biochem 2018;47:1785-1799 \\ \begin{tabular}{l|l|l} 
and BiOChemistry & $\begin{array}{l}\text { DOI: } 10.1159 / 000491060 \\
\text { Published online: June 28, } 2018\end{array}$ & $\begin{array}{l}\text { C } 2018 \text { The Author(s). Published by S. Karger AG, Basel } \\
\text { www.karger.com/cpb }\end{array}$
\end{tabular}}

Hong et al.: Gremlin1 Induced by MSCs Promoted EMT in ESCC

carcinoma than that in normal esophageal tissues. After knocking down the expression of GREM1 in ECa109 and TE-1 cells with a stably integrated lentiviral shRNA, we found that the co-application of MSCs initiated the EMT and enhanced the growth of the esophageal tumors in vivo and that treatment with conditional medium from scrambled-MSCs could enhanced esophageal cancer cell proliferation and invasion in vitro. This effect decreased when the shGREM1-MSCs were co-injected into nude mice or when esophageal carcinoma cells were cultured in the conditional medium from shGREM1-MSCs. Additionally, western blotting results demonstrated that the shGREM1-MSCs-CM-controlled EMT alteration in esophageal carcinoma cells was mediated by the BMP4/TGF- $\beta$ signaling pathway. Overall, this work provided a new strategy and a potential clinical application of GREM1 silencing within MSCs for the treatment of esophageal cancer.

GREM1 is a BMP antagonist and is also related to differentiation. It is overexpressed in various types of human cancers and has been reported to play a role in oncogenesis. In this study, clinical samples of esophageal cancer were obtained, and we found that GREM1 was more highly expressed in esophageal carcinoma than that in normal tissues. This is consistent with publicly available data that GREM1 is highly overexpressed in tumors. One previous study showed that the high expression of GREM1 mRNA was significantly correlated with a bulky tumor, which is one of the most malignant features of cancer [15]. GREM1 has also been found to be secreted from stromal cancer-associated fibroblasts in colon cancer, with its expression localized to invasion fronts [16, 17]. Mulvihill, et al., reported that its overexpression in certain organs, such as the lung, may promote tumor growth, and its down-regulation in other organs, such as the brain, may inhibit tumor growth. Therefore, GREM1 plays a significant role in the pathogenesis of several human cancers.

The tumor microenvironment is variably composed of endothelial cells, immune cells, MSCs, cancer-associated fibroblasts (CAFs), extracellular matrix and soluble factors [18]. CAFs are crucial components of the tumor microenvironment, responsible for the carcinogenesis, proliferation, migration and invasion of cancer cells by secreting various growth factors and cytokines through EMT [19]. Recent evidence suggests that MSCs are selectively located in tumors, where they contribute to the formation of CAFs, and promote tumor growth by differentiating into CAFs. Luo, et al. reported that the EMT and migration of prostate cancer stem cells is driven by CAFs [5]. The invasion and metastasis of tumors has been reported to be dependent on CAF activation, in cancers including breast, prostate, and liver $[5,20]$. In this study, we found that MSCs increased the malignancy of esophageal tumors both in vitro and in vivo through alteration of the EMT, while the downregulation of GREM1 decreased this effect. This work provides direct evidence to support the role of MSCs in esophageal tumor growth and migration through changing the tumor microenvironment.

Increasing evidence is proving an understanding of the pathological mechanisms of esophageal cancer requires attention to the tumor microenvironment. In esophageal cancer, environmental exposure lead to chronic inflammation, promoting survival and proliferation through the constitutive activation of pro-inflammatory signaling pathways [21]. CAFs modulate the microenvironment by communicating with the tumor via secreted factors. The Gremlin gene is known to encode a member of the BMP antagonist family. BMP4 is a member of the TGF- $\beta$ family, GREM1 binds to and forms heterodimers with BMP-4, prevents ligandreceptor interaction and inactivates downstream signaling. Thus, regulation of GREM1 in CAFs is likely to modulate growth and metastasis in tumor cells. Our findings validate the above conclusions, showing that GREM1 downregulation in MSCs could negative affect esophageal tumor growth through reversing the EMT. In this study, we found that GREM1 exerted an inhibitory effect on the BMP4 expression, which may affect the homeostasis between BMP4 and TGF- $\beta$ signaling, leading to enhancement of TGF- $\beta$ expression, as confirmed by western blotting. Therefore, impairment in the BMP4 and enhancement of TGF- $\beta$ induced by GREM1 downregulation in MSCs are two major signal mechanisms necessary to attenuate the malignancy of esophageal tumors. 


\section{Conclusion}

Taken together, our study suggested that GREM1 delivered by MSCs promoted EMT in ESCC in vitro and in vivo, which is partly through TGF- $\beta$ /BMP signaling pathway. The results provide experimental evidence to a potential therapeutic target in the treatment of esophageal cancer.

\section{Abbreviations}

EMT (epithelial-mesenchymal transition); MSCs (mesenchymal stromal cells); TME (tumor microenvironment); qRT-PCR (quantitative reverse transcription-polymerase chain reaction); ESCC (esophageal squamous-cell carcinoma); CCK8 (Cell Counting Kit); GREM1 (Gremlin1); CM (conditioned medium); scrambled-MSCs-CM (conditioned medium from the scrambled MSCs); BMP (bone morphogenetic protein); TGF- $\beta$ (transforming growth factor-beta); shGREM1-MSCs (shRNA silencing of GREM1 in MSCs ); shGREM1-MSCs-CM (conditioned medium from shGREM1-MSCs); BMP (bone morphogenetic protein); ELISA (Enzyme-linked immunosorbent assay ); CCK8 (Cell Counting Kit); CAFs (cancer-associated fibroblasts).

\section{Acknowledgements}

This work was supported by National Natural Science Foundation of China (81770494); Natural Science Foundation of Guangdong Province (2015A030313026, 2017A030313877); Medical Research Foundation of Guangdong Province (B2013098); The Fundamental Research Funds for Colleges and Universities (3161033); Guangdong Province Key Laboratory of Malignant Tumor Epigenetics and Gene Regulation (No. 2017B030314026); and the National College Students' innovation Project (No. 201701102).

QLX and TL designed the study and wrote the manuscript. DXH, YL, LW, HXC and ZZ performed the experiments. DXH, HXC and ZZ collected the data. WJH analyzed and interpreted data. All authors read and approved the final manuscript.

\section{Disclosure Statement}

The authors declare to have no competing interests.

\section{References}

1 Jemal A, Bray F, Center MM, Ferlay J, Ward E, Forman D: Global Cancer Statistics. CA-Cancer J Clin 2011;61:6990.

2 Allen DC: Oesophageal Carcinoma. Ceylon Med J 2013;47:348.

3 Shinya O, Shin'Ichi M, Osamu K, Tomoyuki G, Yusuke A, Manabu M: Recent Advances From Basic and Clinical Studies of Esophageal Squamous Cell Carcinoma. Gastroenterology 2015;149:1700-1715.

4 Thiery JP, Acloque H, Huang RY, Nieto MA: Epithelial-mesenchymal transitions in development and disease. Cell 2009;139:871-890.

5 Wulf GG, Chapuy B, Trümper L: Mesenchymal stem cells from bone marrow. Phenotype, aspects of biology, and clinical perspectives. Med Klin (Munich) 2006;101:408-413.

6 Quail DF, Joyce JA: Microenvironmental regulation of tumor progression and metastasis. Nat Med 2013;19:1423-1437.

7 Ungefroren H, Sebens S, Seidl D, Lehnert H, Hass R: Interaction of tumor cells with the microenvironment. Cell Commu Signal. 2011;9:18. 


\section{Cellular Physiology Cell Physiol Biochem 2018;47:1785-1799

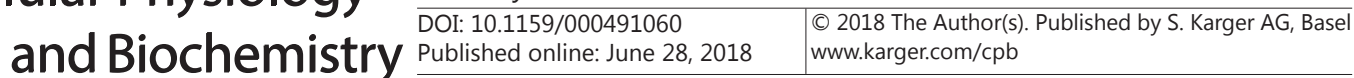

Hong et al.: Gremlin1 Induced by MSCS Promoted EMT in ESCC

8 Whiteside TL: The tumor microenvironment and its role in promoting tumor growth. Oncogene 2008;27:5904-5912.

-9 Pittenger MF, Mackay AM, Beck SC, Jaiswal RK, Douglas R, Mosca JD, Moorman MA, Simonetti DW, Craig S, Marshak DR: Multilineage potential of adult human mesenchymal stem cells. Science 1999;284:143-147.

10 Lazennec G, Jorgensen C: Concise review: adult multipotent stromal cells and cancer: risk or benefit? Stem Cells 2008;26:1387-1394.

11 Hsu DR, Economides AN, Wang XR, Eimon PM, Harland RM: The Xenopus dorsalizing factor gremlin identifies a novel family of secreted proteins that antagonize BMP activities. Mol Cell 1998;1:673-683.

12 Namkoong H, Shin SM, Kim HK, Ha SA, Cho GW, Hur SY, Hur SY, Kim TE, Kim JW: The bone morphogenetic protein antagonist gremlin 1 is overexpressed in human cancers and interacts with YWHAH protein. BMC Cancer 2006;6:74.

13 Li Y, Wang Z, Wang S, Zhao J, Zhang J, Huang Y: Gremlin-mediated decrease in bone morphogenetic protein signaling promotes aristolochic acid-induced epithelial-to-mesenchymal transition (EMT) in HK-2 cells. Toxicology 2012;297:68-75.

14 Xiang Q, Hong D, Liao Y, Cao Y, Liu M, Pang J, Zhou J, Wang G, Yang R, Wang M, Xiang AP: Overexpression of Gremlin1 in Mesenchymal Stem Cells Improves Hindlimb Ischemia in Mice by Enhancing Cell Survival. J Cell Physiol 2017;232:996-1007.

15 Sato M, Kawana K, Fujimoto A, Yoshida M, Nakamura H, Nishida H, Inoue T, Taguchi A, Takahashi J, Adachi K, Nagasaka K, Matsumoto Y, Wada-Hiraike O, Oda K, Osuga Y, Fujii T: Clinical significance of Gremlin 1 in cervical cancer and its effects on cancer stem cell maintenance. Oncol Rep 2015;35:391-397.

16 Karagiannis GS, Musrap N, Saraon P, Treacy A, Schaeffer DF, Kirsch R, Riddell RH, Diamandis EP: Bone morphogenetic protein antagonist gremlin-1 regulates colon cancer progression. Biol Chem 2015;396:163183.

-17 Karagiannis GS, Treacy A, Messenger D, Grin A, Kirsch R, Riddell RH, Diamandis EP. Expression patterns of bone morphogenetic protein antagonists in colorectal cancer desmoplastic invasion fronts. Mol Oncol 2014;8:1240-1252.

18 Hanahan D, Weinberg RA: Hallmarks of cancer: the next generation. Cell 2011;144:646-674.

19 Ogawa M, LaRue AC, Drake CJ: Hematopoietic origin of fibroblasts/myofibroblasts: Its pathophysiologic implications. Blood 2006;108:2893-2896.

20 Kubo N, Araki K, Kuwano H, Shirabe K: Cancer-associated fibroblasts in hepatocellular carcinoma. World J Gastroenterol 2016;22:6841-6850.

21 Lin EW, Karakasheva TA, Hicks PD, Bass AJ, Rustgi AK: The tumor microenvironment in esophageal cancer. Oncogene 2016;35:5337-5349. 